\title{
On the assessment of a tunable auxiliary mass damper with a friction damper in its suspension: numerical study
}

\author{
E.L.C. Guerineau ${ }^{1}$, H.T. Coelho ${ }^{1}$, F.P. Lepore Neto ${ }^{1}$, M.B. Santos ${ }^{1}$ and J. Mahfoud ${ }^{2}$. \\ ${ }^{1}$ Mechanical System Laboratory, Mechanical Engineering School, Federal University of Uberlandia, Brazil. \\ ${ }^{2}$ LaMCoS, Université de Lyon, CNRS, INSA-Lyon, France.
}

\begin{abstract}
Auxiliary Mass Damper's (AMD) performance is susceptible to changes in the frequency or in the excitation force's nature. Therefore, to improve the robustness of the AMD it's necessary to design new systems which are tunable and that could be used over large frequency range. In this work a friction damper, which is an association in series of a spring and a scratcher, is used to tune the AMD at the same time it dissipates the mechanical energy of the principal mass by changing the normal force on the scratcher. Three normal force control strategies, and two combinations of them, are studied: i) The normal force is assumed constant; ii) The normal force is obtained from the solution of the equation of motion assuming null displacement for the principal mass; iii) The normal force is obtained based on the vibratory system's state variables condition, guarantying that the direction of the friction force promotes the movement of the principal mass toward its static equilibrium position. The effectiveness of the proposed tunable AMD, where the adaptability is obtained by controlling the normal force on the smart friction damper, is evaluated based on mass and frequency ratios variations for each strategy.
\end{abstract}

Keywords: Tunable Auxiliary Mass Damper, Variable Damped Absorber, Semi -Active Device Control Strategies, Vibration Attenuation and Friction Damper.

\section{Introduction}

Auxiliary masses are frequently attached to vibrating systems by springs and damping devices to reduce the amplitude of vibration of the system. Depending on the application, these auxiliary mass systems fall into one of two distinct classes. A Dynamic Vibration Absorber (DVA) is an auxiliary mass on a spring, which has a damping factor as lower as possible, once it is tuned to the frequency of the excitation force a system's antiresonance is introduced on this frequency reducing the primary system's vibration amplitudes. And, when it is necessary to provide damping, an auxiliary system is attached to the structure, so that, the auxiliary mass system works as a particular form of damper. This system is called Damped Absorber or Auxiliary Mass Damper (AMD) and it is an extension of the DVA concept [1] .

To improve the AMD's performance some researchers use an active device on its suspension. These devices are active springs, done with memory shape alloys, piezo stacks and other actuators able to tune the AMD to a desired frequency [2]. Other solutions are the semi-active systems, which use friction dampers $[3,4]$ or magnetorheological dampers [5] to tune the frequency of the AMD system and also to dissipate the mechanical energy.

AMD enable reducing vibration amplitudes without energy consumption, within a narrow frequency band for which the AMD has been tuned. Unfortunately, when changes in the excitation nature or in the system parameters occur, its performance will drop drastically. To improve AMD's robustne ss a suitable approach is a semi-active absorber (or adaptive, tunable), which changes its characteristics according to the necessities. Such a device has its physical parameters, as consequence also its impedance, adjustable. Associated to a suitable control law it is possible to adapt the system to different variety of excitations reducing the vibration amplitude. This way, the system becomes a Tunable Auxiliary Mass Damper (TAMD).

The energy necessary to tune the AMD is much less than the energy necessary to achieve the same attenuation using active actuators, once for the active systems the energy is expended to work against the excitation force. 
The aim of this work is to develop a new TAMD where the adeptly is obtained by controlling the normal force of a smart friction damper. The numerical study demonstrates the effectiveness of the developed strategy.

\section{Theoretical approach}

A schema of the system studied is shown on Fig.1. It is a two DOFs that can be modeled as a one Degree of Freedom (DOF) linear vibratory system $\left(m_{1}\right.$, in black) coupled to an AMD using a friction damper $\left(m_{2}\right.$, in red).

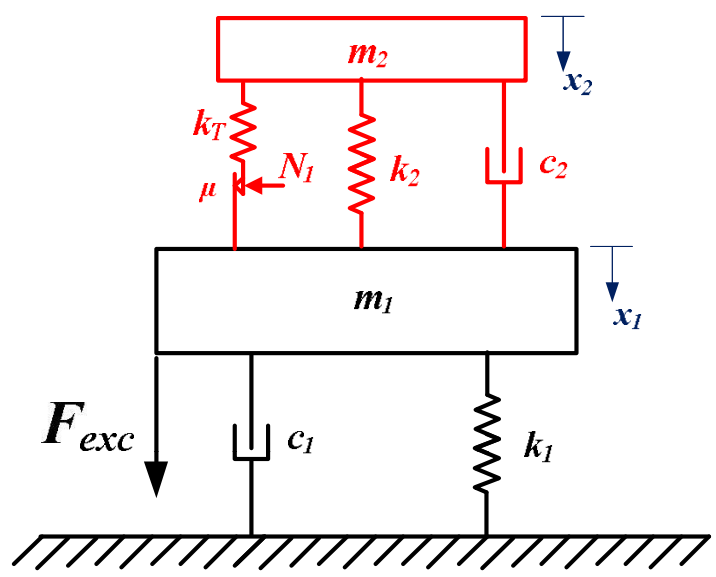

Fig.1 Schema of the system studied.

The adaptability is achieved by using a friction damper (Fig.2). It is an association in series of a spring and a scratcher which will tune the TAMD. This system enables to dissipate the mechanical energy of the principal mass, by changing the normal force on the scratcher, therefore are changed the apparent damping coefficient and stiffness of the TAMD's suspension. The force between nodes (1) and (2), indicated on Fig.2, is $F_{12}$ and can be written as presented on Eq.(1).

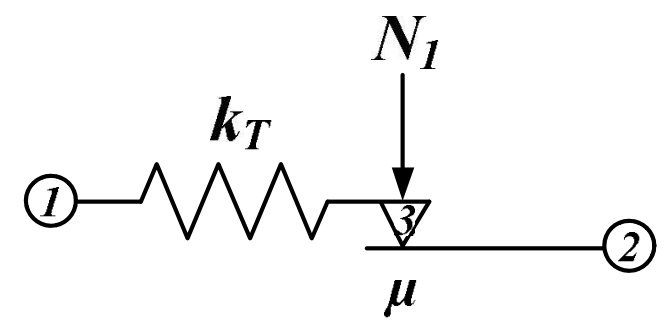

Fig.2 Friction damper model

$F_{12}=\left\{\begin{array}{lll}k_{T}\left(x_{2}-x_{1}\right) & \text { if } & k_{T}\left(x_{2}-x_{1}\right) \leq \mu N_{1} \\ \mu N_{1} & \text { if } & k_{T}\left(x_{2}-x_{1}\right)>\mu N_{1}\end{array}\right.$

Points (1) and (2) from friction damper, as shown on Fig.2, are attached to mass $m_{1}$ and $m_{2}$ of the vibratory system respectively. The TAMD's suspension is composed by the stiffness $k_{2}$ and the damping $c_{2}$, as linear elements, and the nonlinear component characterized by the tangential stiffness $k_{T}$ and the scratcher which has its force as defined in Eq.(1). The suspension between $m_{1}$ and the inertial frame is composed by the stiffness $k_{1}$ and the damping $c_{1}$. The motion equation for the entire system becomes:

$$
\begin{aligned}
& {\left[\begin{array}{cc}
m_{1} & 0 \\
0 & m_{2}
\end{array}\right]\left\{\begin{array}{l}
\ddot{x}_{1} \\
\ddot{x}_{2}
\end{array}\right\}+\left[\begin{array}{cc}
c_{1}+c_{2} & -c_{2} \\
-c_{2} & c_{2}
\end{array}\right]\left\{\begin{array}{l}
\dot{x}_{1} \\
\dot{x}_{2}
\end{array}\right\}+} \\
& {\left[\begin{array}{cc}
k_{1}+k_{2} & -k_{2} \\
-k_{2} & k_{2}
\end{array}\right]\left\{\begin{array}{l}
x_{1} \\
x_{2}
\end{array}\right\}=\left[\begin{array}{c}
1 \\
-1
\end{array}\right] F_{12}+\left[\begin{array}{l}
1 \\
0
\end{array}\right] F_{\text {exc }}}
\end{aligned}
$$

The equation of motion has been integrated using the methodology proposed by Lu et al [3] which, uses the state space formulation from the linear system, and dispose the nonlinear force from friction damper as part of the excitation forces.

Knowing that mass $\left(m_{2} / m_{1}\right)$ and frequency $\left(\omega_{2} / \omega_{1}\right)$ ratios affect DVA's and AMD's behavior, the numerical assessment aimed to determine the best ratios to be used for the future experimental workbench, under design. As mentioned before TAMD works associated to a control law. Five control laws are used, that are three main normal force control strategies, and two combinations of them: i) The normal force is assumed constant [S1]; ii) The normal force is obtained, from Eq.(3), which is the solution of the equation of motion assuming null displacement for the principal mass, i.e. $x_{1}=0$ [S2]; iii) If $F_{12}$ produces a movement of $m_{1}$ in the direction of its static equilibrium position, then the normal force is stemming from Eq.(4), otherwise it is null [S3]. The strategy [S4] uses the same logic described for [S3], but now the normal force is calculated using Eq.(3). And [S5] also uses the logic developed for [S3], however as for [S1] it uses a constant value for $N_{1}$.

$N_{1}=\frac{\left|m_{2} \ddot{x}_{2}+c_{2} \dot{x}_{2}+k_{2} x_{2}\right|}{\mu}$
$N_{1}=\frac{k_{T}\left(x_{2}-x_{1}\right)}{\mu}$

To compare all methodologies it is necessary to establish a criteria that enable to show the reduction of the resonance amplitude peak simultaneously to the reduction of the amplitude of the receptance over the entire interested frequency band. It is clear from the literature that DVA split the original resonant peak in two resonant peaks, which, can be disastrous to the vibratory system if the excitation force contains harmonics with these new resonant frequencies. Normally, two parameters are used simultaneously to describe the performance of the control systems: Maximum Value ( $L_{\infty}$ norm) and the $L_{2}$ norm. The former indicates the maximum amplitude expected for the system response and the second the overall mean value of the response. A similar statement is used in the control technique $H_{\infty} / H_{2}$ [6]. Therefore, the best control law performance will provide the lowest values for both norms. In this work the performance parameter $\left(P_{p}\right)$ is defined as:

$P_{p}=\frac{\text { maximum receptance amplitude }}{\text { receptance amplitude norm }}$

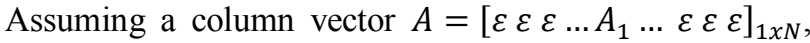
where $\varepsilon$ is a real constant closest to zero and $A_{1}$ a real positive constant, which represents the receptance with one peak only on the frequency spectrum $\left(A_{1}=\delta\left(f_{1}\right)\right.$, 
where $\delta\left(f_{1}\right)$ is the Dirac function on frequency $\left.f_{1}\right)$, the parameter $P_{p}$ can be written as:

$P_{p}=\lim _{\varepsilon \rightarrow 0} P_{p}=\lim _{\varepsilon \rightarrow 0} \frac{A_{1}}{\sqrt{(N-1) \varepsilon^{2}+A_{1}^{2}}}=\frac{A_{1}}{A_{1}}=1$

The other extreme is a constant amplitude receptance, which are represented by the column vector $A=$ $\left[\begin{array}{lllllll}\varepsilon & \varepsilon & \varepsilon & \varepsilon & \varepsilon & \varepsilon\end{array}\right]_{1 x N}$ for this the parameter $P_{p}$ is written as:

$P_{p}=\lim _{\varepsilon \rightarrow 0} P_{p}=\lim _{\varepsilon \rightarrow 0} \frac{\varepsilon}{\sqrt{N \varepsilon^{2}}}=\frac{1}{\sqrt{N}}$

Equations (6) and (7) give the maximum and minimum of the performance parameters as defined on Eq. (5).

\section{Numerical results}

To obtain a reasonable comparison among the obtained results and those of the literature, mass and frequency ratios are the same described by Harris and Piersol (2002). In this work the ratios of mass used are $m_{2} / m_{1}=$ $[0.1,0.2,0.3,0.4,0.5]$ and for the frequency are $\omega_{2} /$ $\omega_{1}=[0.1,0.5,1]$. These ratios with the five control strategies will lead to 75 combinations of results.

The numerical results presented at this section has been obtained using the following physical parameters value, which represent the parameters from a designed modification of the experimental workbench used on previously works [7,8], $m_{1}=3.0 \mathrm{~kg}, c_{1}=20 \mathrm{Ns} / \mathrm{m}$ and $k_{1}=68 \mathrm{kN} / \mathrm{m}$ for the vibratory system and the physical parameters for the secondary system $\left(m_{2}, c_{2}\right.$ and $\left.k_{2}\right)$ are deduced from the mass and frequency ratios in use. The contact parameters are the tangential stiffness $k_{T}=260 \mathrm{k}$ $N / m$ and the friction coefficient $\mu=0.3$. It is important to write that these parameters are theoretical ones, and should be estimated as soon as the test rig has been constructed.

To obtain the aforementioned receptances all strategies are applied for the control of the 2 DOF composed vibratory system excited with a $F_{e x c}=5 N$ harmonic force. The excitation was harmonic with its frequency sweeping from $5 \mathrm{~Hz}$ up to $100 \mathrm{~Hz}$, in steps of $1 \mathrm{~Hz}$. The stead state response was obtained using 10 periods, and the steady state has been assumed to occur after 10 periods of oscillation. These parameters have been chosen after an observation of the system response at the resonance. For the strategies which uses constant normal force value the applied value was $N_{1}=10 \mathrm{~N}$.

The values of the performance parameter $P_{p}$ for each combination, for the 1 DOF free vibration and for the well-tuned DVA are shown on Figure 3. On this figure the color bar indicates the value of $P_{p}$ and the arrows indicates the location of the best result for each strategy, also for the location of the DVA and the 1DOF free vibration.

Figure 4 presents the receptances, which give the lowest $P_{p}$ values for each strategy. These are compared with the receptances for $1 \mathrm{DOF}$ and for DVA.

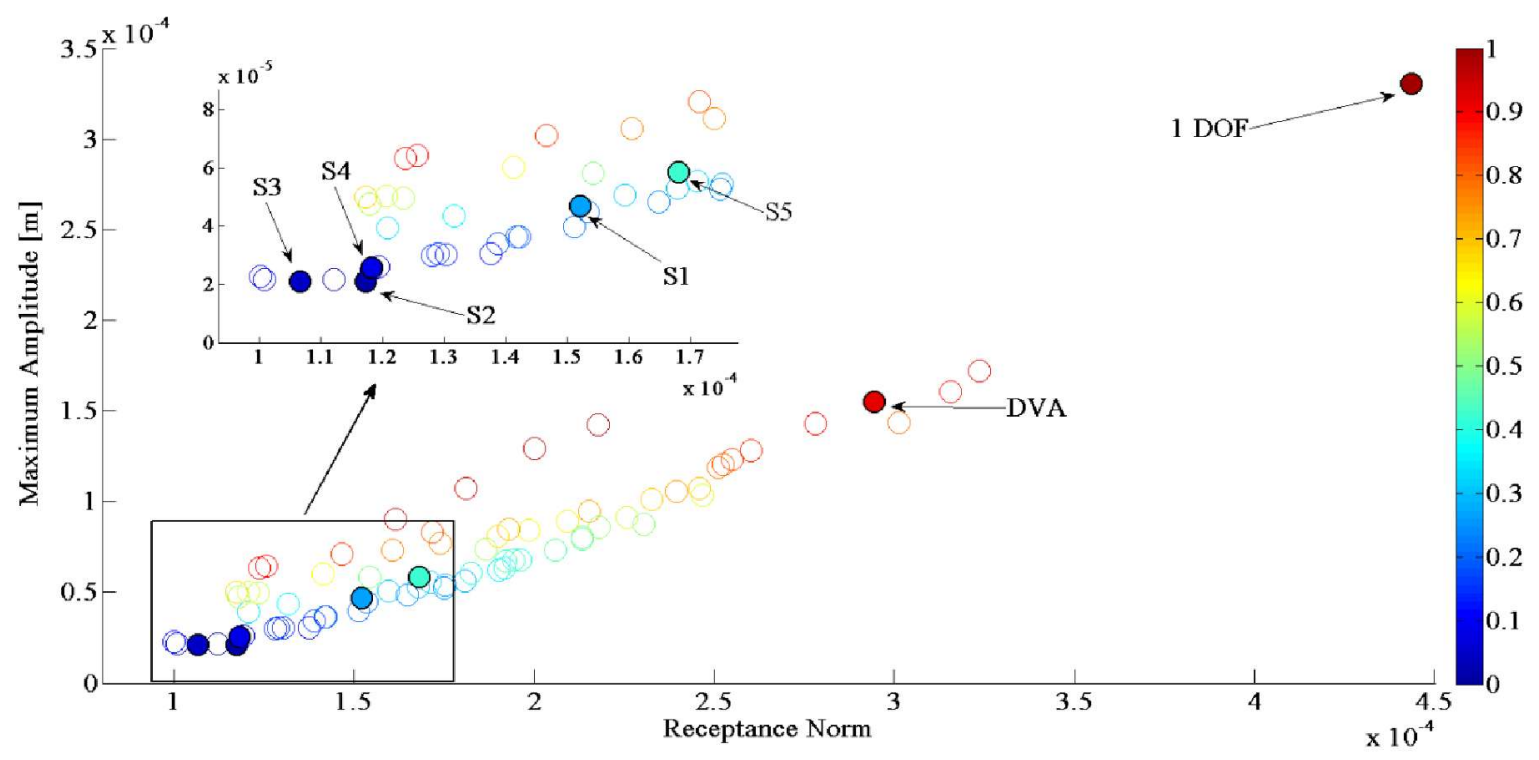

Fig.3 Maximum amplitude and receptance norm chart.

Table 1 summarizes the mass and frequency ratios combination which results in the lowest values for $P_{p}$ for each strategy. Also presents the ratios used to tune the DVA and their respective performance parameter value. The 1 DOF free vibration have had its receptance also quantified by using the performance parameter $P_{p}$.

All strategies promote an improvement in the attenuation of the resonant peaks as well as in the $L_{2}$ norm value over the analyzed frequency band. The best performance parameter is for Strategy S2, in fact, its receptance does not have any resonant peak and maintains the low value over the other frequency bands when compared to the 1 DOF curve. Strategies S3 and S4 give similar results when compared to $\mathrm{S} 2$, their highest values for $P_{p}$ are due the maximum amplitude of the receptance which are 
higher than S2 maximum amplitude. It should be noticed that all strategies give better results than DVA.

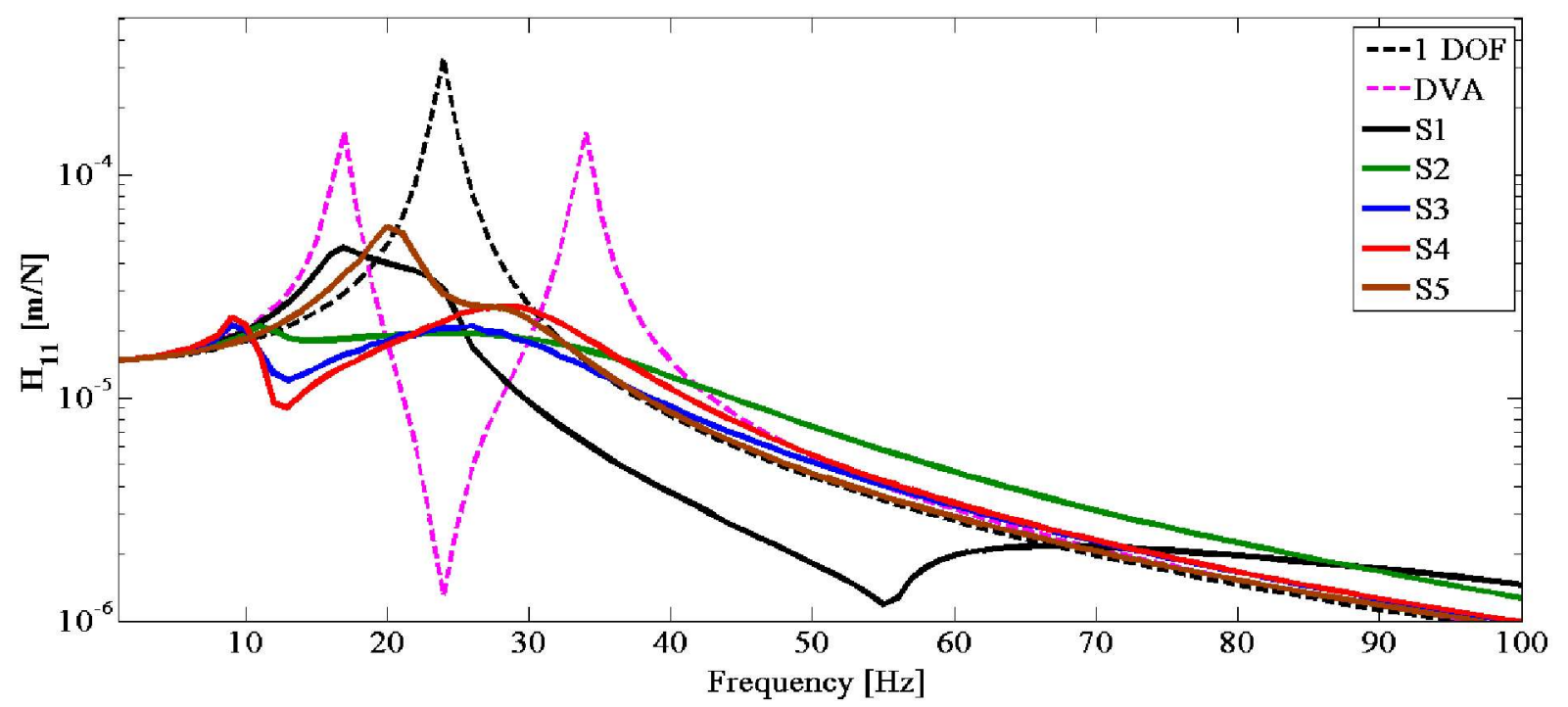

Fig.4 Receptance results.

Table 1 Ratios combination and $P_{p}$ value for the bests of each strategy.

\begin{tabular}{|c|c|c|c|}
\hline \multirow{2}{*}{ CONTROL STRATEGY } & \multicolumn{2}{|c|}{ RATIOS COMBINATION } & \multirow{2}{*}{$\boldsymbol{P}_{\boldsymbol{p}}$} \\
\cline { 2 - 4 } & $\boldsymbol{\omega}_{\mathbf{2}} / \boldsymbol{\omega}_{\mathbf{1}}$ & $\boldsymbol{m}_{\mathbf{2}} / \boldsymbol{m}_{\mathbf{1}}$ & 0.74 \\
\hline 1 DOF & $(-)$ & $(-)$ & 0.52 \\
\hline DVA & 1.0 & 0.5 & 0.30 \\
\hline STRATEGY S1 & 0.5 & 0.5 & 0.17 \\
\hline STRATEGY S2 & 0.5 & 0.3 & 0.19 \\
\hline STRATEGY S4 & 0.5 & 0.4 & 0.21 \\
\hline STRATEGY S5 & 0.5 & 0.5 & 0.34 \\
\hline
\end{tabular}

\section{Conclusions}

A performance parameter has been defined and was shown to be effective to identify the receptance with the lowest peak and the lowest $L_{2}$ norm over the frequency band of analysis. Using the numerical simulations is possible to affirm that the proposed TAMD model presents better results than the traditional DVA. Future works, already undergoing, will confirm the numerical results experimentally.

\section{Acknowledgements}

The authors are grateful to the agencies and bureaus which had been supported this research project: Capes, Fapemig, CNPq and Brafitec.

\section{References}

1. HARRIS, C.M. and PIERSOL, A.G. Harris' Shock and Vibration Handbook. Fifth Edition. McGraw-Hill HANDBOOKS, 2002.

2. CHATTERJEE, S., Optimal active absorber with internal state feedback for controlling resonant and transient vibration, Journal of Sound and Vibration, 329, p. 5397-5414, 2010.

3. LU, L.-Y.; CHUNG, L. -L.; WU, L.Y. and LIN, G. -L. Dynamic Analysis of Structures with Friction Devices Using Discrete-Time State-Space
Formulation. Computers and Structures. Taiwan, v. 84, p. 1049-1071, 2006.

4. LIN, C.-C, LIN, G.-L. and WANG, J.-F., Protection of seismic structures using semi-active friction TDM, Earthquake Engineering and Structural Dynamics, 39, p. 635-659, 2010.

5. WEBER, F., Semi-active vibration absorber based on real-time controlled MR damper, Mechanical Systems and Signal Processing, 46, p. 272-288, 2014.6. ROTEA, M.A. and KHARGONEKAR, P.P., Mixed $\mathrm{H}^{2}$-optimal Control with an $\mathrm{H}^{\infty}$ constraint: The State Feedback Case, Automatica, 27(2): p. 307-316, 1991.

6. ROTEA, M.A. and KHARGONEKAR, P.P., Mixed $\mathrm{H}^{2}$-optimal Control with an $\mathrm{H}^{\infty}$-constraint: The State Feedback Case, Automatica, 27(2): p. 307-316, 1991.

7. COELHO, H.T., SANTOS, M.B. and LEPORE NETO, F.P. Control Strategies for Friction Dampers: Numerical Assessment and Experimental Investigations. Second International Brazilian Conference on Tribology - TriboBR 2014, p.1-11, 2014.

8. COELHO, H.T., SANTOS, M.B., LEPORE NETO, F.P and J. MAHFOUD. Control strategies for friction dampers: numerical assessment and experimental investigations. MATEC Web of Conferences 16, 07007, p.1-4, 2014 